\title{
Experimental investigation of the behaviour of tungsten and molybdenum alloys at high strain-rate and temperature
}

\author{
Martina Scapin $^{1, \text { a }}$, Claudio Fichera ${ }^{1}$, Federico Carra ${ }^{1,2}$, and Lorenzo Peroni ${ }^{1}$ \\ ${ }^{1}$ Politecnico di Torino, Mechanical and Aerospace Engineering Department \\ ${ }^{2}$ CERN, Engineering Department, Mechanical \& Materials Engineering Group
}

\begin{abstract}
The introduction in recent years of new, extremely energetic particle accelerators such as the Large Hadron Collider (LHC) gives impulse to the development and testing of refractory metals and alloys based on molybdenum and tungsten to be used as structural materials. In this perspective, in this work the experimental results of a tests campaign on Inermet $R$ IT180 and pure Molybdenum (sintered by two different producers) are presented. The investigation of the mechanical behaviour was performed in tension varying the strain-rates, the temperatures and both of them. Overall six orders of magnitude in strain-rate (between $10^{-3}$ and $10^{3} \mathrm{~s}^{-1}$ ) were covered, starting from quasi-static up to high dynamic loading conditions. The high strain-rate tests were performed using a direct Hopkinson Bar setup. Both in quasi-static and high strain-rate conditions, the heating of the specimens was obtained with an induction coil system, controlled in feedback loop, based on measurements from thermocouples directly welded on the specimen. The temperature range varied between 25 and $1000{ }^{\circ} \mathrm{C}$. The experimental data were, finally, used to extract the parameters of the Zerilli-Armstrong model used to reproduce the mechanical behaviour of the investigated materials.
\end{abstract}

\section{Introduction}

The introduction in recent years of new, extremely energetic particle accelerators such as the Large Hadron Collider (LHC) required the development of advanced methods to predict the behaviour of components, such as the Beam Intercepting Devices (BID), in case of direct beam impact. For this reasons some components of the LHC machine are designed to operate in harsh radioactive environment highly solicited from thermostructural point of view. As a matter of fact, they can directly interact with high energy beam and, in case of accident, the energy deposition on the bulk material could be potentially destructive. The impact produces a sudden energy deposition in the components generating outgoing of shock-waves and deforming the material in very high strain-rate and temperature conditions $[1,2]$. In order to study and predict the impact effects, the numerical simulations of the materials response via FE codes could be fundamental [3]. In the same way, they could represent the reference point for the design of complex experimental tests directly performed in the accelerator [4]. Obviously, reliable predictions need that the materials behaviour is described through confident constitutive relationships.

This context gives impulse to the development and testing of refractory metals and alloys based on molybdenum, tungsten, rhenium and iridium to be used as structural materials. In the scientific literature, few works can be found in which the mechanical characterization of these materials was performed in a wide range of temperature and strain-rate (e.g. [5-7]). Moreover, not

\footnotetext{
${ }^{a}$ Corresponding author: martina.scapin@polito.it
}

always a strength model calibration was performed, which otherwise it is fundamental in FE simulations.

In this perspective, in this work the experimental

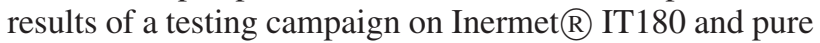
molybdenum are presented. Given the extreme loading conditions in which the materials could operate (e.g. thermo-mechanical shocks in accident situations), the investigation of the mechanical behaviour was performed in a wide range of strain-rates and temperatures. Finally, the experimental data are analyzed and used to extract the parameters of the Zerilli-Armstrong model [8].

\section{Materials under investigation}

The materials under investigation are a tungsten heavyalloy and pure molybdenum.

The tungsten alloy is INERMET R IT180 (IT180 in the following), supplied by Plansee, which is actually used in the collimation system of the Large Hadron Collider at CERN. The material has a nominal composition of $95 \mathrm{wt} \%$ $\mathrm{W}, 3.5 \mathrm{wt} \% \mathrm{Ni}$ and $1.5 \mathrm{wt} \% \mathrm{Cu}$. As it is possible to see from the microstructure of Fig. 1, the $\mathrm{W}$ grains are quite big, with an average dimension of about $100 \mu \mathrm{m}$, and are surrounded by the binder phase. The second phase is a $\mathrm{W}-\mathrm{Ni}-\mathrm{Cu}$ mixture, has a low melting point, represents the ductile connection between the brittle $\mathrm{W}$ grains and, finally, provides the necessary thermal and electrically continuity to the material. The material behaviour in terms of flow stress and damage is a consequence of the complex interaction between the behaviour of the two phases, depending on the values of strain-rate and temperature. The material could show different types of failure modes [7]: tungsten-tungsten grain boundary separation, tungsten 


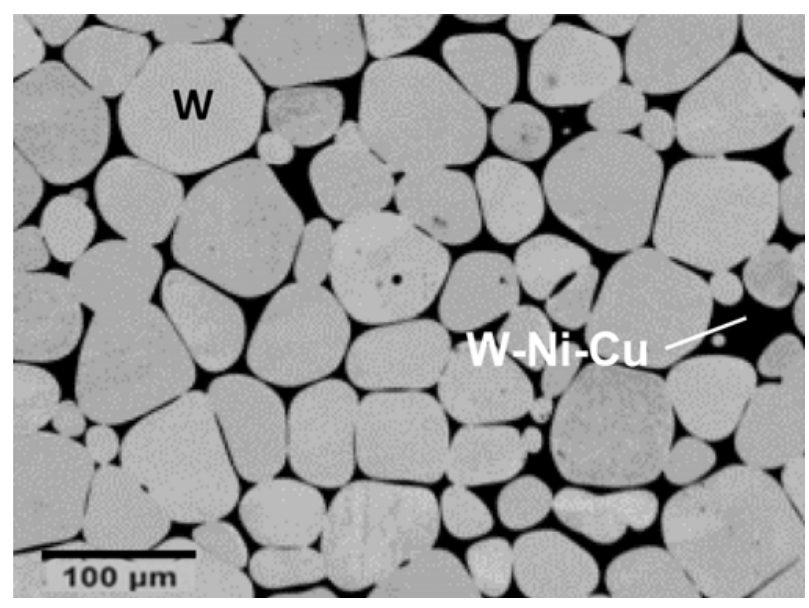

Figure 1. IT180 microstructure.

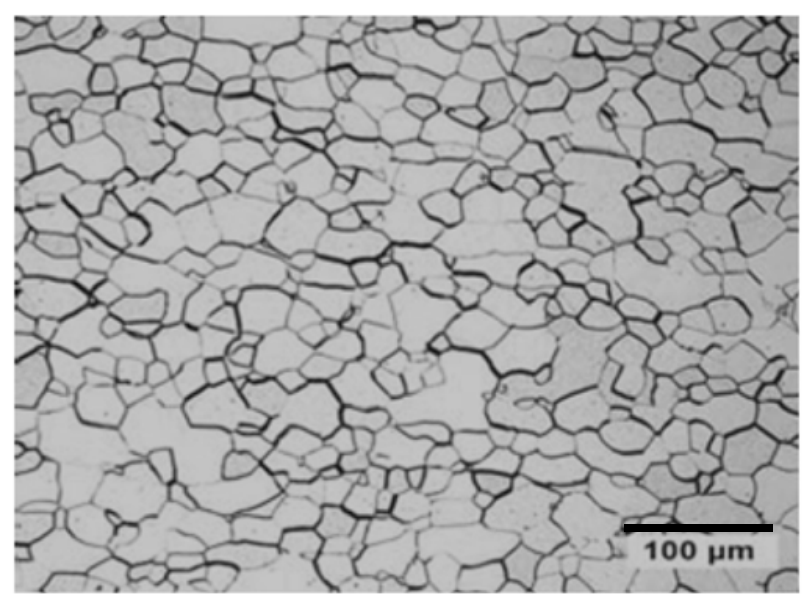

Figure 2. Pure molybdenum microstructure (Mo1).

grain cleavage, tungsten-binder interfacial separation and matrix fracture.

In Fig. 2, the microstructure of pure molybdenum is reported. The material is obtained starting from finegrained metal powders of high purity which are pressed, sintered, hot worked and finally annealed to obtain a regular grain structure.

In more details, two different materials are analyzed, which nominally differ only for the producer: one is supplied by Plansee (in the following Mo1) and the second one by AT\&M (in the following Mo2).

\section{Experimental tests}

The mechanical characterization was performed both in tension and compression, but in this work the attention is focused on the results obtained in tension. The specimen has a gage length of $5 \mathrm{~mm}$ and a diameter of $3 \mathrm{~mm}$ [9].

A series of tests at room temperature at different strainrates is performed in order to obtain information about the strain and strain-rate sensitivity of the material. The strainrate sensitivity is investigated starting from $10^{-3} \mathrm{~s}^{-1}$ up to $10^{1} \mathrm{~s}^{-1}$. For all the materials, the test at $10^{3} \mathrm{~s}^{-1}$ is not performed since in this condition the material is too brittle and no reliable results can be obtained. The mediumlow strain-rate test are performed using electro-mechanical and servo-hydraulic testing machines. The high strain-rate condition is reached by means of a Hopkinson Bar setup in direct configuration.

In addition, a series of tests at different temperatures in both quasi-static and high strain-rate loading conditions is performed in order to obtain information about the thermal softening of the material. The atmosphere of the tests is air: this is not a problem for IT180, which is not subjected to heavy oxidation, while for molybdenum inert atmosphere should be used. The temperature range varies between $25^{\circ} \mathrm{C}$ and $800^{\circ} \mathrm{C}$ in quasi-static loading conditions, while temperatures up to $1000^{\circ} \mathrm{C}$ are investigated at high strainrate. The difference is mainly due to the fact that at low strain-rate the test duration is too high and the testing equipment could not withstand a high temperature for a long time; on the other hand, the dynamic test duration is about $400 \mu \mathrm{s}$.

The experimental setups and the testing methodology are widely described in [9].

For each testing condition at least three repetitions are performed in order to control the data scattering, then the average curves is reported in Figs. 3-5. The results are shown in terms of engineering stress vs. engineering strain curves at different loading conditions.

The evaluation of the data scatter is performed calculating the mean standard deviation for each loading condition, as reported in Table 1 . The repeatability of the tests is good for the three materials, even if Mo2 is the material with the highest dispersion.

Looking at the results in terms of engineering stress vs. strain, it is possible to conclude that both IT180 alloy and pure molybdenum are temperature and strain-rate sensitive. Moreover the behaviour is such expected for BCC materials: the yield stress is a function of temperature and strain-rate and this justify the choice of the Z-A model.

Analyzing in more detail the IT180 results, it is possible to notice that the elongation at failure dramatically decreases increasing the strain-rate, while at high temperature (both in quasi-static and dynamic regimes), it grows increasing the temperature, reaches a maximum and then decreases again for very high temperature. Moreover, the tests are characterized by the absence of necking, which allows to analytically analyze the results. At high strain-rate, a temperature of $200^{\circ} \mathrm{C}$ is needed for performing the test.

For what concerns the two grades of pure molybdenum, it is possible to notice that, in general, both the materials exhibit some yielding instabilities. The two materials have a similar behaviour, even if a higher stability at very high temperature is found for Mo2. Increasing the temperatures (both in quasi-static and dynamic cases), the materials strength decreases, showing a sort of plateau effect starting from $400^{\circ} \mathrm{C}$. In the same manner, also the elongation at failure increases. Increasing the strain-rate the materials harden and reduce the elongation at failure.

At high strain-rate, a temperature of $100{ }^{\circ} \mathrm{C}$ is sufficient for performing the test. Starting from medium-high strainrate (above $10^{1} \mathrm{~s}^{-1}$ ), over a certain amount of deformation, the plastic work becomes relevant as well as the heat generated inside the specimen. In this condition, the 

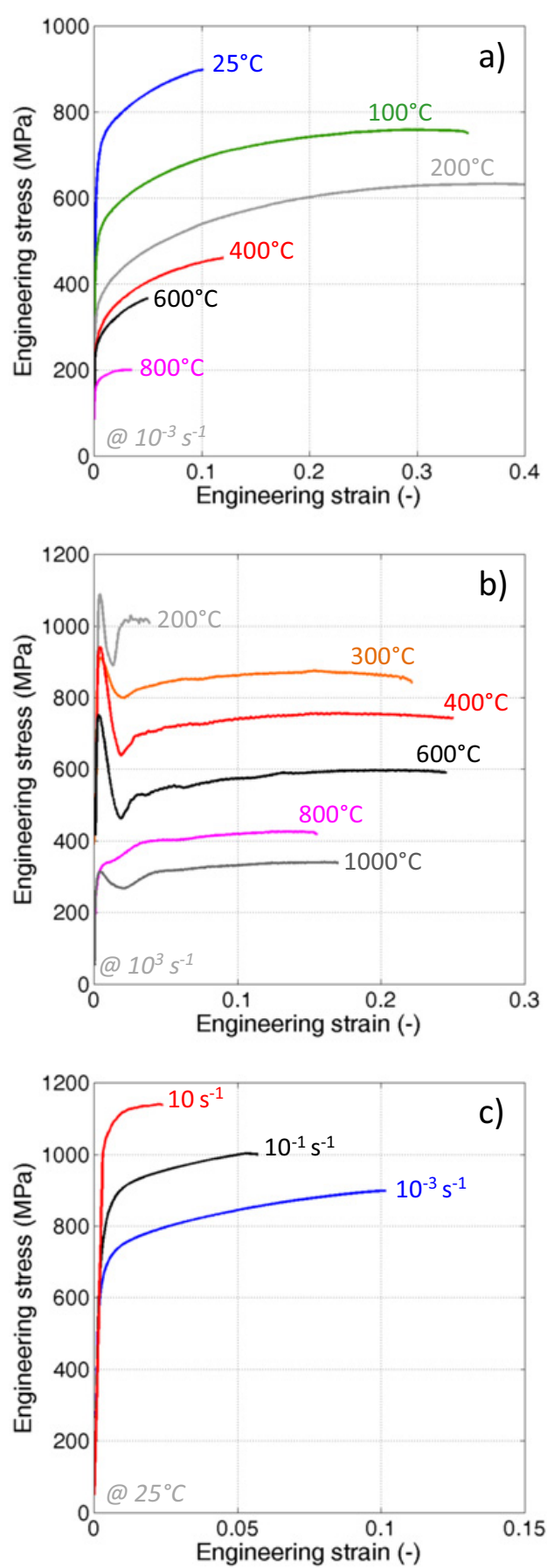

Figure 3. IT180 - Engineering stress vs. strain curves: a) quasistatic loading condition varying the temperature; b) dynamic loading condition varying the temperature and c) tests at room temperature varying the strain-rate.

thermal softening balances and overcomes the effect of the strain-rate hardening: the deformation process starts to be adiabatic.

\section{Data analysis}

The experimental results showed that the materials are strongly dependent on strain, strain-rate and temperature. For these reasons, it is important to find an appropriate
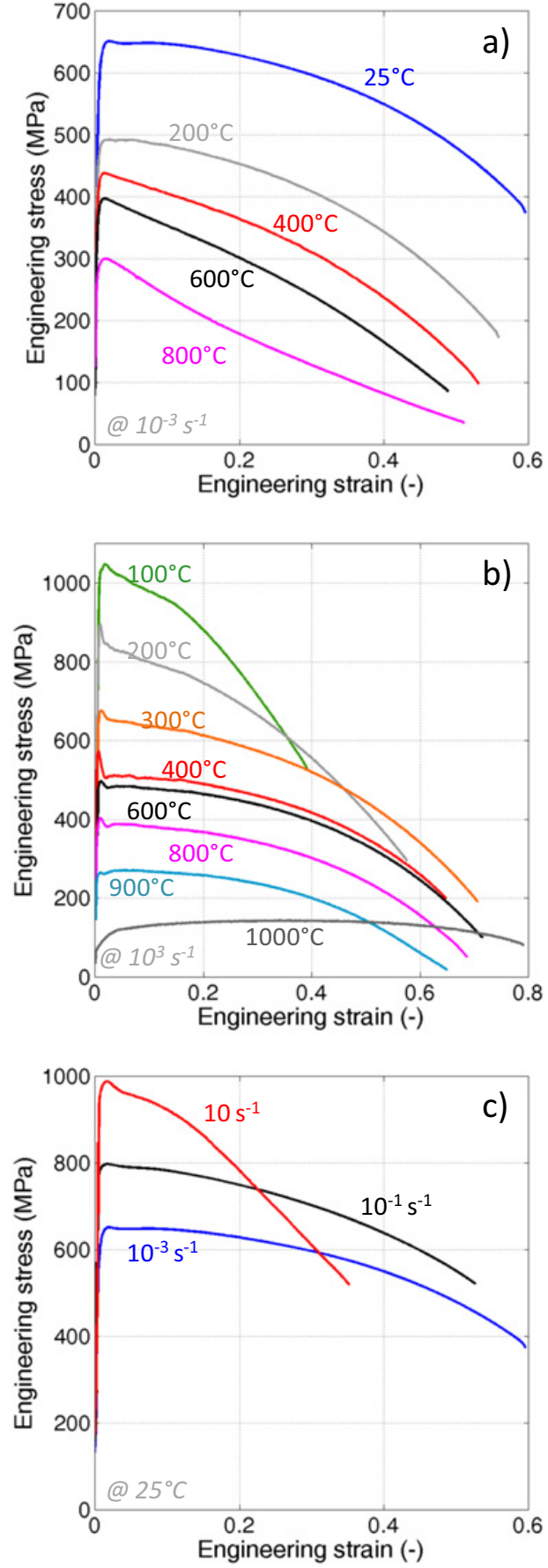

Figure 4. Mo1 - Engineering stress vs. strain curves: a) quasistatic loading condition varying the temperature; b) dynamic loading condition varying the temperature and c) tests at room temperature varying the strain-rate.

material model for the mechanical behaviour description and a suitable strategy for the parameters identification.

As it is well known, in the scientific literature, it is possible to find a great number of constitutive models for the viscoplastic flow description. One of the simplest models able to take into account a coupling effect between temperature and strain-rate and which is suitable for BCC materials is the Zerilli-Armstrong model. It expresses the flow stress as a function of the plastic strain, the strain-rate 

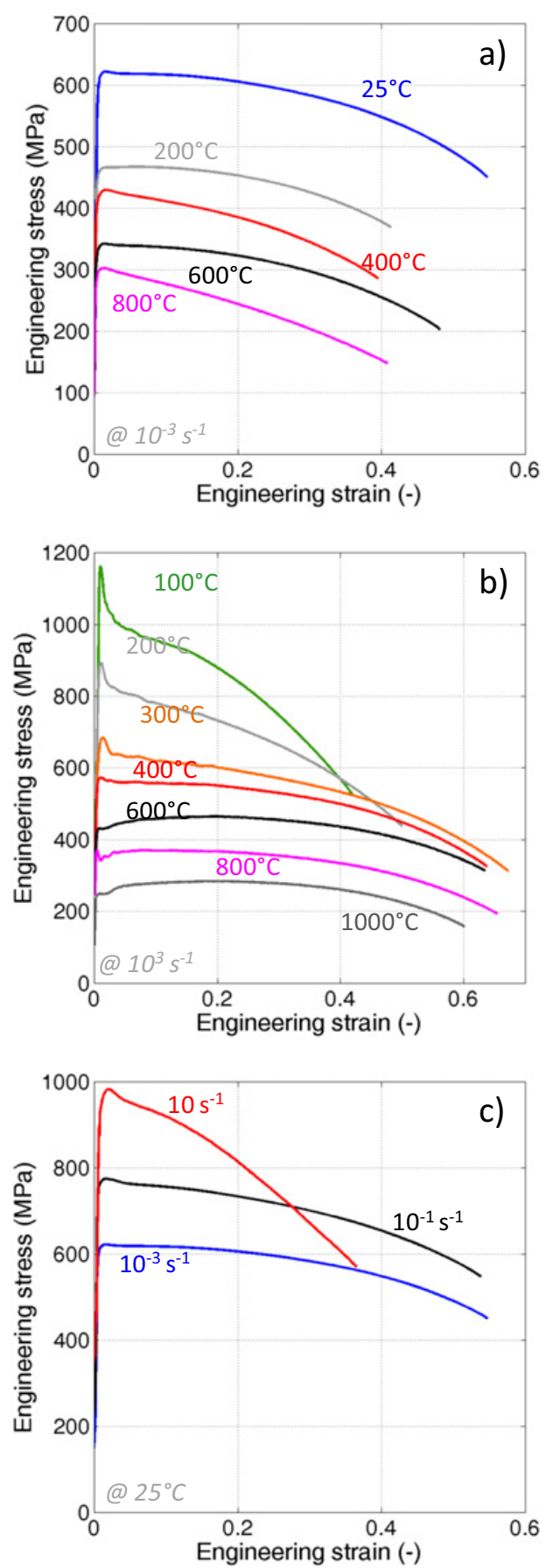

Figure 5. Mo2 - Engineering stress vs. strain curves: a) quasistatic loading condition varying the temperature; b) dynamic loading condition varying the temperature and c) tests at room temperature varying the strain-rate.

and the absolute temperature:

$$
\sigma_{y}=C_{1}+C_{2} e^{\left(-C_{3}+C_{4} \ln \dot{\varepsilon}\right) T}+C_{5} \varepsilon^{n}
$$

in which $C_{i}$ are the material model parameters. In accordance with the original formulation, some of these parameters could be related to physical quantities of the material, but in this work all of them are considered as optimization variables. In more detail, they are obtained via a multi-objective optimization process, in
Table 1. Data scatter for IT180, Mo1 and Mo2 in terms of mean standard deviation (MPa).

\begin{tabular}{|c|c|c|c|}
\hline & IT180 & Mo1 & Mo2 \\
\hline $10^{-3} \mathrm{~s}^{-1} @ 25^{\circ} \mathrm{C}$ & 11 & 7 & 25 \\
\hline $\mathbf{1 0}^{-3} \mathbf{s}^{-1} @ 100^{\circ} \mathrm{C}$ & 2 & - & - \\
\hline $10^{-3} \mathrm{~s}^{-1} @ 200^{\circ} \mathrm{C}$ & 3 & 8 & 26 \\
\hline $10^{-3} \mathrm{~s}^{-1} @ 400{ }^{\circ} \mathrm{C}$ & 12 & 10 & 33 \\
\hline $10^{-3} \mathrm{~s}^{-1} @ 600^{\circ} \mathrm{C}$ & 10 & 9 & 24 \\
\hline $10^{-3} \mathrm{~s}^{-1} @ 800^{\circ} \mathrm{C}$ & 11 & 27 & 8 \\
\hline $10^{-1} \mathbf{s}^{-1} @ 25^{\circ} \mathrm{C}$ & 15 & 10 & 28 \\
\hline $10^{1} \mathrm{~s}^{-1} @ 25^{\circ} \mathrm{C}$ & 7 & 22 & 6 \\
\hline $10^{3} \mathrm{~s}^{-1} @ 100^{\circ} \mathrm{C}$ & - & 11 & 7 \\
\hline $10^{3} \mathrm{~s}^{-1} @ 200^{\circ} \mathrm{C}$ & 18 & 11 & 30 \\
\hline $10^{3} \mathrm{~s}^{-1} @ 300^{\circ} \mathrm{C}$ & 14 & 14 & 20 \\
\hline $10^{3} \mathrm{~s}^{-1} @ 400^{\circ} \mathrm{C}$ & 19 & 4 & 31 \\
\hline $10^{3} \mathrm{~s}^{-1} @ 600^{\circ} \mathrm{C}$ & 2 & 7 & 14 \\
\hline $10^{3} s^{-1} @ 800^{\circ} \mathrm{C}$ & 10 & 16 & 20 \\
\hline $10^{3} \mathrm{~s}^{-1} @ 900^{\circ} \mathrm{C}$ & - & 36 & - \\
\hline $10^{3} s^{-1} @ 1000$ & 3 & 25 & 9 \\
\hline
\end{tabular}

Table 2. Optimized parameters of the Z-A model for the three materials.

\begin{tabular}{|l|l|l|l|l|l|l|}
\hline & $\begin{array}{l}C_{\mathbf{1}} \\
(\mathrm{MPa})\end{array}$ & $\begin{array}{l}C_{\mathbf{2}} \\
(\mathrm{MPa})\end{array}$ & $\begin{array}{l}C_{\mathbf{3}} \\
\left(10^{-3} / \mathrm{K}\right)\end{array}$ & $\begin{array}{l}C_{\mathbf{4}} \\
\left(10^{-4} / \mathrm{K}\right)\end{array}$ & $\begin{array}{l}C_{\mathbf{5}} \\
(\mathrm{MPa})\end{array}$ & $\begin{array}{l}n \\
(-)\end{array}$ \\
\hline IT180 & 21 & 2572 & 3.416 & 1.591 & 915 & 0.497 \\
\hline Mo1 & 0 & 4030 & 6.449 & 3.073 & 524 & 0.110 \\
\hline Mo2 & 0 & 2833 & 5.203 & 2.639 & 485 & 0.135 \\
\hline
\end{tabular}

which at the same time the data coming from different loading conditions are simultaneously used as targets. The optimization is based on the minimisation of the sum of the single normalized mean square errors.

Due to the different plastic behaviour of IT180 and molybdenum, two different approaches are followed.

In case of IT180, an analytical approach is used, in which the data fitting is performed on true stress vs. true plastic strain curves calculated from the engineering ones (under the hypothesis of volume conservation). The range of applicability in strain is limited by the instability strain (maximum of the engineering stress vs. strain curves), which is sufficient in case of IT180. A series of different solutions are analyzed and good results, in terms of correlation between experimental and predicted responses, are obtained when the experimental data at temperature over $400{ }^{\circ} \mathrm{C}$ in quasi-static condition are omitted from the optimization process. In the optimization all the available repetitions are taken into account for the determination of 6 unknown (a more in-depth description of the procedure is reported in [9]). The optimized parameters are summarized in Table 2.

On the other hand, in case of Mo1 and Mo2, a numerical inverse approach is used. The parameters identification is reached varying the material strength parameters of the FE models (one for each experimental loading and constraint condition) and comparing the model results with the experimental data with the aim to obtain the best correlation. The great advantage of this procedure is that no hypothesis about the internal specimen stress-strain, temperature or strain-rate fields is made: in fact, the comparison is made in terms of macroscopic 
Table 3. Percentage Normalized Mean Square Error for IT180, Mo1 and Mo2 obtained by the comparison between target and computed curves ("nc" means that data of the corresponding loading conditions is not considered in the optimization).

\begin{tabular}{|c|c|c|c|}
\hline & IT180 & Mo1 & Mo2 \\
\hline $10^{-3} \mathrm{~s}^{-1} @ 25^{\circ} \mathrm{C}$ & 0.0342 & 0.0492 & 0.0582 \\
\hline $1^{-3} \mathrm{~s}^{-1} @ 100^{\circ} \mathrm{C}$ & 0.0339 & - & - \\
\hline $10^{-3} s^{-1} @ 200{ }^{\circ} \mathrm{C}$ & 0.0424 & $\mathrm{nc}$ & $\mathrm{nc}$ \\
\hline $10^{-3} s^{-1} @ 400{ }^{\circ} \mathrm{C}$ & 2.357 & $\mathrm{nc}$ & $\mathrm{nc}$ \\
\hline $10^{-3} s^{-1} @ 600{ }^{\circ} \mathrm{C}$ & $\mathrm{nc}$ & $\mathrm{nc}$ & $\mathrm{nc}$ \\
\hline $10^{-3} \mathrm{~s}^{-1} @ 800{ }^{\circ} \mathrm{C}$ & $\mathrm{nc}$ & $\mathrm{nc}$ & $\mathrm{nc}$ \\
\hline $10^{-1} s^{-1} @ 25^{\circ} \mathrm{C}$ & 0.00310 & 0.0404 & 0.129 \\
\hline $\mathbf{1 0}^{1} \mathrm{~s}^{-1} @ 25^{\circ} \mathrm{C}$ & 0.0729 & 0.463 & 1.400 \\
\hline $10^{3} \mathrm{~s}^{-1} @ 100^{\circ} \mathrm{C}$ & - & 0.256 & 0.358 \\
\hline $10^{3} \mathrm{~s}^{-1} @ 200^{\circ} \mathrm{C}$ & 0.147 & 0.140 & 0.0868 \\
\hline $10^{3} \mathrm{~s}^{-1} @ 300^{\circ} \mathrm{C}$ & 0.0266 & 0.0728 & 0.321 \\
\hline $10^{3} \mathrm{~s}^{-1} @ 400^{\circ} \mathrm{C}$ & 0.0412 & 0.527 & 0.0540 \\
\hline $10^{3} \mathrm{~s}^{-1} @ 600^{\circ} \mathrm{C}$ & 0.0267 & 0.772 & 0.276 \\
\hline $10^{3} \mathrm{~s}^{-1} @ 800^{\circ} \mathrm{C}$ & 0.733 & 0.400 & 0.268 \\
\hline $10^{3} \mathrm{~s}^{-1} @ 900^{\circ} \mathrm{C}$ & - & $\mathrm{nc}$ & - \\
\hline $10^{3} \mathrm{~s}^{-1} @ 1000{ }^{\circ} \mathrm{C}$ & 2.94 & nc & $\mathrm{nc}$ \\
\hline
\end{tabular}

quantities which, in general, are force and displacement. The optimization of the parameters is performed with a dedicated algorithm included in the software LS-OPT.

Also for molybdenum, a series of different solutions are analyzed and the best results, in terms of correlation between experimental and predicted responses, are obtained when the experimental data at different temperatures in quasi-static condition are omitted from the optimization process, as well as the dynamic experimental data at temperature over than $800^{\circ} \mathrm{C}$. Each optimization process has 9 objectives and 5 variables to be determined $\left(C_{1}\right.$ is set equal to 0 ). The target curves are obtained as the average behaviour of each loading condition. The optimized parameters are summarized in Table 2.

For the three materials, the qualitative comparison between experimental and computed quantities is reported in Fig. 6 for the dynamic tests at high strain-rate. As it is possible to notice, the optimized Z-A models are able to reproduce the materials behaviours with a good level of accuracy.

A more quantitative indication of the error is reported in Table 3 in terms of percentage Normalized Mean Square Error (NMSE), defined by the relationship:

$$
N M S E=\frac{1}{n} \sum_{k=1}^{n}\left(\frac{x_{k}-\hat{x}_{k}}{\max (x)}\right)^{2} \times 100
$$

where the index $k$ identifies one of the $n$ points of the objective (target curve), $x$ represents the experimental target and $\hat{x}$ represents the computed response.

In Fig. 7 the Z-A models obtained for the three materials are compared. In Fig. $7 \mathrm{a}$ the models are compared in terms of flow stress curves in quasi-static loading condition at room temperature. In Fig. $7 \mathrm{~b}$, the comparison is made in terms of equivalent stress as a function of temperature at $5 \%$ of strain. As expected, the models obtained for the two grades of molybdenum produce very similar results. For these materials, the quasistatic behaviour varying the temperature is extrapolated.
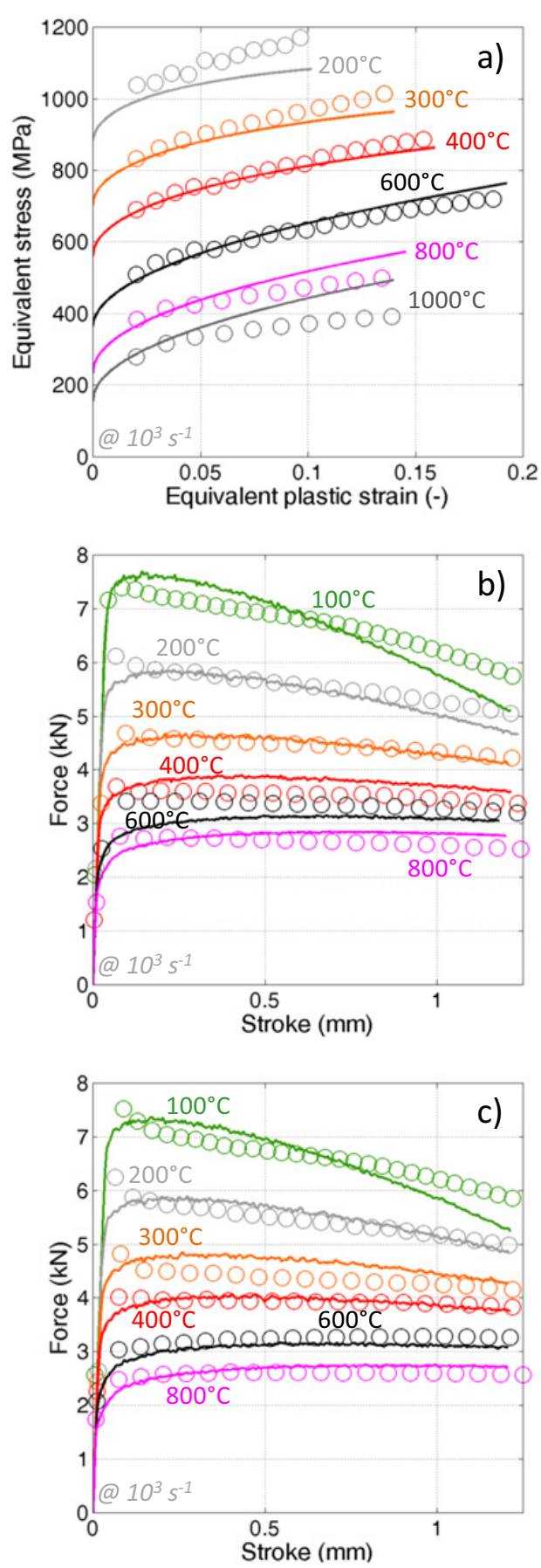

Figure 6. Comparison between experimental and computed quantities: a) for IT180 (analytical optimization); b) and c) for Mo1 and Mo2, respectively (numerical inverse optimization).

For what concerns the alloy IT180, the comparison reveals that the material shows a higher strength with respect to molybdenum at low temperatures, while the opposite is expected increasing the temperature, as a consequence of the presence of the second low-melting phase.

\section{Conclusions}

In this work an experimental test campaign was performed in order to study the strain-rate and temperature sensitivities of a tungsten alloy (IT180) and pure 

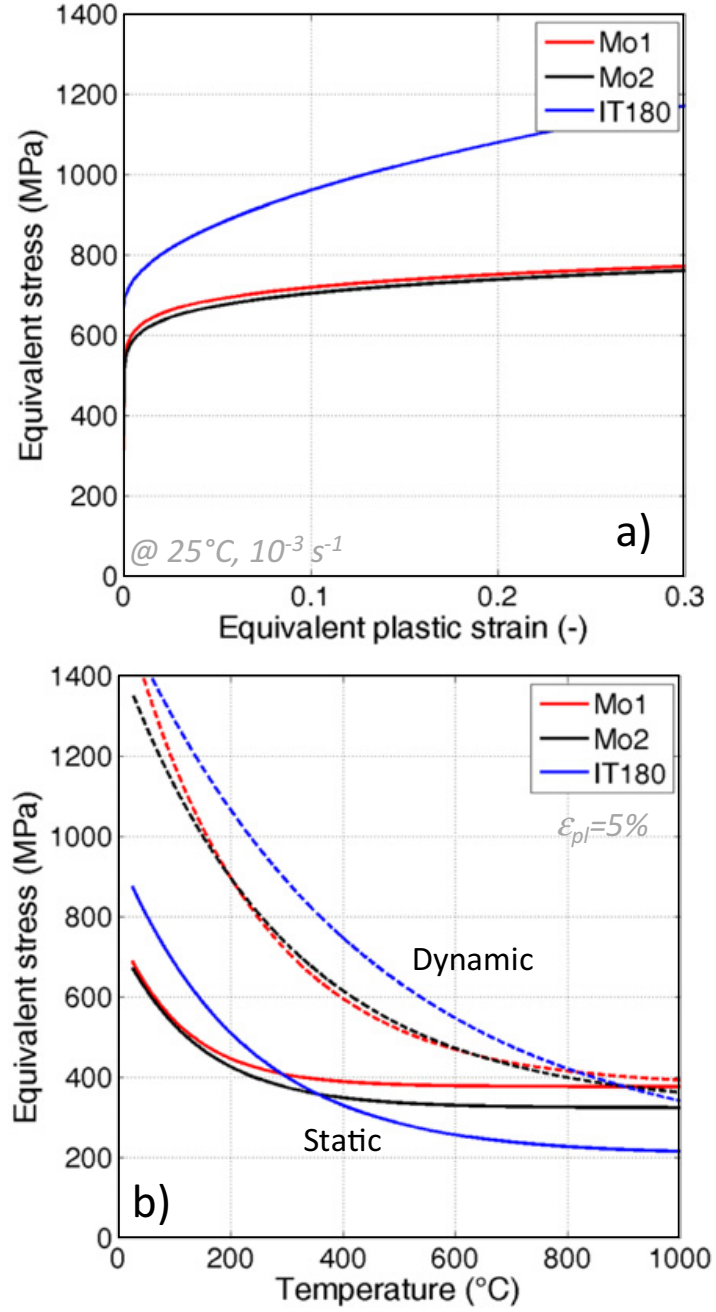

Figure 7. Comparison between optimized Z-A models obtained for the three materials: a) in terms of plastic flow stress in quasistatic loading condition at room temperature; b) in terms of equivalent stress as a function of temperature in quasi-static and dynamic cases.

molybdenum (in two different grades). The tests were performed in tension starting from quasi-static up to high strain-rate loading conditions. The dynamic tests were performed using a direct Hopkinson bar setup. The temperature sensitivity was investigated from room temperature up to $1000^{\circ} \mathrm{C}$ both in quasi-static and dynamic regimes. The heating of the specimen was obtained by an induction coil system controlled in closed loop by means of thermocouples directly welded on the specimens. The results shown the materials were both strain-rate and temperature sensitive. The IT180 was characterized by the absence of necking with respect to molybdenum. All the materials exhibited a decrease of the elongation at failure increasing the strain-rate such that no reliable results were obtained from dynamic tests at room temperature. The experimental data were used to extract the coefficients for the Z-A model, used in the BCC formulation. The parameter identification was based on the definition of a multi-objective optimization. Two different approaches were used. In case of IT180 an analytical fitting was performed on the true stress vs. true plastic strain. On the other hand, due to instability and necking, in case of molybdenum, a numerical inverse approach was used. The optimized Z-A models were able to reproduce the materials behaviour with a sufficient level of accuracy. Comparing the results, it was possible to notice that the predicted results were in good agreement with the experimental one. The Z-A models obtained for the two grades of molybdenum produced very similar results, while the model obtained for IT180 predicted a higher strength at low temperatures associated to a more significant reduction in strength increasing the temperature.

\section{References}

[1] A. Bertarelli et al., "Limits for beam induced damage: reckless or too cautious?" in Proceeding of Workshop on LHC performance, Chamonix 2011.

[2] M. Scapin, L. Peroni and A. Dallocchio, J. Nucl. Mater. 420 (1-3) pp. 463-472 (2012).

[3] M. Scapin, L. Peroni, V. Boccone, F. Cerutti, Comput. Struct. 141 pp. 74-83 (2014).

[4] A. Bertarelli et al., Nucl. Instrum. Meth. B 308 pp88-99 (2013).

[5] G. P. Skoro, J. R. J. Bennett, T. R. Edgecock, C. N. Booth, J. Nucl. Mater. 426 pp. 45-51 (2012).

[6] S. Nemat-Nasser, W. Guo, M. Liu, Scripta Mater. 40 (7) pp. 859-872 (1999).

[7] W-S. Lee, G-L. Xiea, C-F. Lin, Mater. Sci. Eng. A 257 pp. 256-267 (1998).

[8] R. W. Armstrong, F. J. Zerilli, J. Phys. C3 (9) pp. 529 534 (1988).

[9] M. Scapin, Int. J. Refract. Met. H. 50 pp. 258-268 (2015). 